TRANSACTIONS OF THE

AMERICAN MATHEMATICAL SOCIETY

Volume 361, Number 12, December 2009, Pages 6549-6565

S 0002-9947(09)04719-9

Article electronically published on July 16, 2009

\title{
F-THRESHOLDS OF HYPERSURFACES
}

\author{
MANUEL BLICKLE, MIRCEA MUSTAŢǍ, AND KAREN E. SMITH
}

\begin{abstract}
We use the $D$-module theoretic description of generalized test ideals to show that in any $F$-finite regular ring the $F$-thresholds of hypersurfaces are discrete and rational. Furthermore we show that any limit of $F$-pure thresholds of principal ideals in bounded dimension is again an $F$-pure threshold; hence in particular the limit is rational.
\end{abstract}

\section{INTRODUCTION}

In characteristic zero one can define invariants of singularities using all divisors over the ambient variety. A key result that makes these invariants computable says that they can be determined by the divisors on a resolution of singularities. For example, if $\mathfrak{a}$ is a sheaf of ideals on a nonsingular variety, then to every nonnegative real number $\lambda$ one associates the multiplier ideal $\mathcal{J}\left(\mathfrak{a}^{\lambda}\right)$. The jumping exponents of $\mathfrak{a}$ are those $\lambda$ such that $\mathcal{J}\left(\mathfrak{a}^{\lambda}\right) \neq \mathcal{J}\left(\mathfrak{a}^{\lambda^{\prime}}\right)$ for every $\lambda^{\prime}<\lambda$. It is an easy consequence of the formula giving the multiplier ideals of $f$ in terms of a log resolution of singularities that the jumping exponents form a discrete set of rational numbers. See for example $\mathrm{Laz}$, Ch. 9 for the basic facts about multiplier ideals and their jumping exponents.

In positive characteristic Hara and Yoshida defined in [HY] an analogue of the multiplier ideals, the (generalized) test ideals. The definition works in a very general setting, involving a notion of tight closure for pairs. In this paper, however, we assume that we work in a regular ring $R$ of characteristic $p>0$ that is $F$-finite, i.e. such that the Frobenius morphism $F: R \rightarrow R$ is finite. If $\mathfrak{a}$ is an ideal in $R$ and if $\lambda$ is a nonnegative real number, then the corresponding test ideal is denoted by $\tau\left(\mathfrak{a}^{\lambda}\right)$. In this context we say that $\lambda$ is an $F$-jumping exponent (or an $F$-threshold) if $\tau\left(\mathfrak{a}^{\lambda}\right) \neq \tau\left(\mathfrak{a}^{\lambda^{\prime}}\right)$ for every $\lambda^{\prime}<\lambda$. The following is our main result about $F$-jumping exponents in positive characteristic.

Theorem 1.1. If $R$ is an $F$-finite regular ring, and if $\mathfrak{a}=(f)$ is a principal ideal, then the F-jumping exponents of $\mathfrak{a}$ are rational and they form a discrete set.

The discreteness and the rationality of $F$-jumping numbers has been proved in BMS for every ideal when the ring $R$ is essentially of finite type over an $F$-finite

Received by the editors July 23, 2007 and, in revised form, January 2, 2008.

2000 Mathematics Subject Classification. Primary 13A35; Secondary 14B05.

Key words and phrases. $F$-thresholds, test ideals, $F$-modules, nonstandard extension.

Partial support was provided by grant SFB/TR 45 of the DFG (first author), NSF grants DMS-0758454, DMS 0111298 and a Packard Fellowship (second author), and NSF grant DMS0500823(third author). 
field. We also mention that for $R=k \llbracket x, y \rrbracket$, with $k$ a finite field, the above result has been proved in $\mathrm{Ha}$ using a completely different approach.

We stress that the difficulty in attacking this result does not come from the fact that there is no available resolution of singularities in positive characteristic. Even in cases when such a resolution is known to exist, the $F$-jumping exponents are not simply given in terms of the numerical information of the resolution. We refer to [MTW for a discussion of the known and conjectural connections between the invariants in characteristic zero and those in characteristic $p$.

In order to prove Theorem 1.1 it is enough to show that the set of $F$-jumping exponents is discrete. The rationality statement follows as in [BMS]: it is enough to use the fact that if $\lambda$ is an $F$-jumping exponent, then so are the fractional parts of $p^{e} \lambda$, for all $e \geq 1$. Moreover, we will see that it is enough to prove the result in the case when $R$ is local.

The crucial step in the proof of the theorem relies on showing that if $\alpha$ is a rational number, then $\alpha$ is not an accumulation point of $F$-jumping exponents of $f$ (irrational $\alpha$ 's are excluded by an inductive argument). The key point in this step is that (after preparing $\alpha$ ) we may rephrase the statement that " $\alpha$ is not an accumulation point of $F$-jumping exponents" as the statement that "a certain element $e_{\alpha}$ of a certain $D_{R}$-module $M_{\alpha}$ (which can be thought of as the $D_{R}$-module generated by $\frac{1}{f^{\alpha}}$; see the paragraph before Lemma 2.3 ) is a $D_{R}$-generator of $M_{\alpha}$ " (Corollary 2.8). Here $D_{R}$ denotes the ring of all differential operators of $R$. This $D_{R}$-module reformulation is an extension of an argument due to Alvarez-Montaner, Blickle and Lyubeznik from AMBL (one can interpret the main result in loc. cit. as the case $\alpha=1$ when $M_{\alpha}=R_{f}$ ). Since we may assume that $R$ is local, one then finishes the argument as in loc. cit. by using the fact that $M_{\alpha}$ has finite length as a $D_{R}$-module (see [Lyu) to conclude that $e_{\alpha}$ indeed generates $M_{\alpha}$ as a $D_{R}$-module (Theorem 2.11). This argument is carried out in detail in Section 2 where also the necessary background and notation is recalled.

The second half of the paper deals with limits of $F$-pure thresholds. We apply our rationality result for formal power series to deduce that every such limit is a rational number. Recall that the $F$-pure threshold of $\mathfrak{a}$ is the smallest (positive) $F$-jumping exponent of $\mathfrak{a}$. This invariant has been introduced by Takagi and Watanabe in TW] who pointed out the analogy with the log canonical threshold in characteristic zero. In a fixed characteristic $p$, we consider the set $\mathcal{T}_{n}$ consisting of all $F$-pure thresholds of principal ideals in regular $F$-finite rings of characteristic $p$ and dimension $\leq n$. We consider also the set $\mathcal{T}_{n}^{\circ}$ of $F$-pure thresholds at the origin for polynomials $f \in k\left[x_{1}, \ldots, x_{n}\right]$, where $k$ is an algebraically closed field of characteristic $p$ (the definition does not depend on $k$ ). It is easy to see that every element in $\mathcal{T}_{n}$ can be computed as the $F$-pure threshold of a formal power series $f$, and therefore it is the limit of the $F$-pure thresholds of the various truncations of $f$. Conversely, we show that every limit of $F$-pure thresholds in bounded dimension is the $F$-pure threshold of some formal power series.

Theorem 1.2. For every prime $p>0$ and every $n \geq 1$, the set $\mathcal{T}_{n}$ is the closure of $\mathcal{T}_{n}^{\circ}$. In particular, every limit of $F$-pure thresholds of principal ideals in F-finite regular rings of bounded dimension is a rational number.

The proof of Theorem 1.2 uses nonstandard methods to construct a power series whose $F$-pure threshold is the limit of a given sequence of $F$-pure thresholds. The necessary background for Theorem 1.2 and its proof are given in Section 3, In the 
final section, Section 4, we record some peculiar features of $F$-pure thresholds and test ideals, and we state some open problems in analogy with some well-known conjectures in birational geometry.

For an application of nonstandard techniques to the study of log canonical thresholds, see $\mathrm{dFM}$. In that case the nonstandard argument is more involved due to the fact that the definition of the log canonical threshold is "less elementary". While the $F$-pure threshold is a more subtle invariant than the log canonical threshold, its definition is "simpler", and this pays off when using nonstandard extensions.

We believe that exploiting the connections and analogies between the invariants in positive and zero characteristic can be very fruitful. For example, results on test ideals such as the Subadditivity and the Restriction Theorems are much easier to prove than for multiplier ideals, and they imply their characteristic zero counterpart by reduction $\bmod p$. Moreover, there are results on multiplier ideals that so far have been proved only by reduction to characteristic $p$ (see the work of Takagi [Ta1] and [Ta2]). On the other hand, certain phenomena that are well understood (or just conjectural) in characteristic zero can point to interesting phenomena in positive characteristic.

\section{Discreteness and Rationality}

We start by reviewing the definition and some basic properties of the generalized test ideals from [BMS]. Let $R$ be a regular ring of characteristic $p>0$. We assume that $R$ is $F$-finite, that is, the Frobenius morphism $F: R \rightarrow R, F(u)=u^{p}$ is finite. Note that $F$-finiteness is preserved by taking quotients, localization and completion (see Example 2.1 in [BMS]). Moreover, if $R$ is $F$-finite, then so are $R[x]$ and $R \llbracket x \rrbracket$.

For an ideal $J$ and for $e \geq 1$, we put $J^{\left[p^{e}\right]}=\left(u^{p^{e}} \mid u \in J\right)$. If $\mathfrak{b}$ is an arbitrary ideal in $R$, then we denote by $\mathfrak{b}^{\left[1 / p^{e}\right]}$ the (unique) minimal ideal $J$ such that $\mathfrak{b} \subseteq J^{\left[p^{e}\right]}$.

Suppose now that $\mathfrak{a}$ is a fixed ideal in $R$ and $\lambda$ is a positive real number. For every $e \geq 1$ we have

$$
\left(\mathfrak{a}^{\left\lceil\lambda p^{e}\right\rceil}\right)^{\left[1 / p^{e}\right]} \subseteq\left(\mathfrak{a}^{\left\lceil\lambda p^{e+1}\right\rceil}\right)^{\left[1 / p^{e+1}\right]},
$$

where $\lceil u\rceil$ denotes the smallest integer $\geq u$. This sequence of ideals stabilizes since $R$ is Noetherian, and the test ideal is defined as $\tau\left(\mathfrak{a}^{\lambda}\right):=\left(\mathfrak{a}^{\left\lceil\lambda p^{e}\right\rceil}\right)^{\left[1 / p^{e}\right]}$ for $e \gg 0$.

Note that if $\lambda>\mu$, then $\tau\left(\mathfrak{a}^{\lambda}\right) \subseteq \tau\left(\mathfrak{a}^{\mu}\right)$. It is shown in [BMS] that for every $\lambda$ there is $\varepsilon>0$ such that

$$
\tau\left(\mathfrak{a}^{\lambda}\right)=\tau\left(\mathfrak{a}^{\lambda^{\prime}}\right)
$$

for every $\lambda^{\prime} \in[\lambda, \lambda+\varepsilon)$. A positive $\lambda$ is called an $F$-jumping exponent of $\mathfrak{a}$ if $\tau\left(\mathfrak{a}^{\lambda}\right) \neq \tau\left(\mathfrak{a}^{\lambda^{\prime}}\right)$ for every $\lambda^{\prime}<\lambda$. It is convenient to make the convention that 0 is an $F$-jumping exponent, too.

If $S$ is a multiplicative system in $R$, then $\tau\left(\left(S^{-1} \mathfrak{a}\right)^{\lambda}\right)=S^{-1} \tau\left(\mathfrak{a}^{\lambda}\right)$. Similarly, if $R$ is local and $\widehat{R}$ is its completion, then $\tau\left((\mathfrak{a} \widehat{R})^{\lambda}\right)=\tau\left(\mathfrak{a}^{\lambda}\right) \widehat{R}$. In particular, if $\lambda$ is an $F$-jumping exponent for $S^{-1} \mathfrak{a}$ or for $\mathfrak{a} \widehat{R}$, then it also has to be an $F$-jumping exponent for $\mathfrak{a}$.

Using the identification of $F$-jumping exponents as $F$-thresholds, one shows in BMS that if $\lambda$ is an $F$-jumping exponent, then so is $p \lambda$. Alternatively, this follows from a strengthening of the Subadditivity Theorem in this context (see Proposition 4.1 below and the remark following it). 
From now on we specialize to the case of a principal ideal $\mathfrak{a}=(f)$. In this case it is shown in BMS that for every $\lambda \geq 1$ we have $\tau\left(f^{\lambda}\right)=f \cdot \tau\left(f^{\lambda-1}\right)$. This implies that if $\lambda \geq 1$, then $\lambda$ is an $F$-jumping exponent of $f$ if and only if $\lambda-1$ is such an exponent.

Combining the above two properties, it follows that if $\lambda$ is an $F$-jumping exponent for $f$, then the fractional parts $\left\{p^{e} \lambda\right\}$ are also $F$-jumping exponents for all $e \geq 1$. Hence if we know that the $F$-jumping exponents of $f$ are discrete, $\lambda$ has to be rational.

Lemma 2.1. If $\lambda=\frac{m}{p^{e}}$ for some positive integer $m$, then $\tau\left(f^{\lambda}\right)=\left(f^{m}\right)^{\left[1 / p^{e}\right]}$.

Proof. By definition, we have $\tau\left(f^{\lambda}\right)=\left(f^{m p^{e^{\prime}-e}}\right)^{\left[1 / p^{e^{\prime}}\right]}$ for some $e^{\prime} \geq e$. Therefore it is enough to show that for every $g \in R$ and every $\ell \geq 1$ we have $\left(g^{p}\right)^{\left[1 / p^{\ell+1}\right]}=g^{\left[1 / p^{\ell}\right]}$. This in turn follows from the flatness of the Frobenius morphism: for an ideal $J$, we have $g \in J^{\left[p^{\ell}\right]}$ if and only if $g^{p} \in J^{\left[p^{\ell+1}\right]}$.

We now recall some basic facts about $R\left[F^{e}\right]$-modules and $D_{R}$-modules. For details we refer to [Lyu or [Bli]. Since $R$ is an $F$-finite regular ring, the ring of differential operators $D_{R} \subseteq \operatorname{End}_{\mathbb{F}_{p}}(R)$ admits the following description. For every $e \geq 0$, let $D_{R}^{e}=\operatorname{End}_{R^{p}}(R)$; hence $D_{R}^{0}=R$. We have $D_{R}^{e} \subseteq D_{R}^{e+1}$ and

$$
D_{R}=\bigcup_{e \in \mathbb{N}} D_{R}^{e} .
$$

By definition, $R$ has a canonical structure of a left $D_{R}$-module. Note also that if $S$ is a multiplicative system in $R$, then we have a canonical isomorphism $S^{-1}\left(D_{R}^{e}\right) \simeq D_{S^{-1} R}^{e}$. The following lemma is a concrete special case of so-called Frobenius descent (see AMBL for a fast introduction), which states that the Frobenius functor induces an equivalence of the category of $R$-modules and $D_{R}^{e}$-modules. In this explicit case it shows the relevance of $D_{R}^{e}$-modules in our setting.

Lemma 2.2. The $D_{R}^{e}$-submodules of $R$ are the ideals of the form $J^{\left[p^{e}\right]}$ for some ideal J. In particular, for every $\mathfrak{b}$ the ideal $\left(\mathfrak{b}^{\left[1 / p^{e}\right]}\right)^{\left[p^{e}\right]}$ is equal to the $D_{R}^{e}$-submodule generated by $\mathfrak{b}$.

Proof. By definition, if $P \in D_{R}^{e}$ and $a, b \in R$, then $P\left(a^{p^{e}} b\right)=a^{p^{e}} P(b)$. This implies that every ideal of the form $J^{\left[p^{e}\right]}$ is a $D_{R^{e}}^{e}$-submodule of $R$.

Conversely, suppose that $I$ is such a submodule, and let $J=\left\{a \in R \mid a^{p^{e}} \in I\right\}$. We clearly have $J^{\left[p^{e}\right]} \subseteq I$, and we show that equality holds. If $\mathfrak{q}$ is a prime ideal in $R$, then $J_{\mathfrak{q}}=\left\{b \in R_{\mathfrak{q}} \mid b^{p^{e}} \in I_{\mathfrak{q}}\right\}$ and $\left(J^{\left[p^{e}\right]}\right)_{\mathfrak{q}}=\left(J_{\mathfrak{q}}\right)^{\left[p^{e}\right]}$. Since $I_{\mathfrak{q}}$ is a $D_{R_{\mathfrak{q}}}^{e}$ submodule of $R_{\mathfrak{q}}$, it follows that it is enough to prove that $I=J^{\left[p^{e}\right]}$ when $R$ is local. Hence we may assume that $R$ is free (and finitely generated) over $R^{p^{e}}$.

If $u_{1}, \ldots, u_{N}$ give a basis of $R$ over $R^{p^{e}}$, then we get morphisms $P_{i}: R \rightarrow R$ that are $R^{p^{e}}$-linear by mapping $u=\sum_{i=1}^{N} a_{i}^{p^{e}} u_{i}$ to $a_{i}^{p^{e}}$. It follows that if $u \in I$, then $P_{i}(u)=a_{i}^{p^{e}} \in I$ for every $i$; hence $a_{i} \in J$, and we have $u \in J^{\left[p^{e}\right]}$.

We denote by $R^{e}$ the $R$ - $R$-bimodule on $R$, with the left structure being the usual one and the right one being induced by the $e^{\text {th }}$ composition of the Frobenius morphism $F^{e}: R \rightarrow R$. We use the scheme-theoretic notation for an extension of scalars via $F^{e}$ : if $M$ is an $R$-module, then we denote by $F^{e *} M$ the $R$-module 
$R^{e} \otimes_{R} M$. We have a canonical isomorphism $R^{e} \otimes_{R} R^{e^{\prime}} \simeq R^{e+e^{\prime}}$ that takes $a \otimes b$ to $a b^{p^{e}}$.

The ring $R[F]$ is the noncommutative ring extension of $R$ generated by a variable $F$ such that $F a=a^{p} F$ for every $a \in R$. For every $e \geq 1$ we also consider the subring $R\left[F^{e}\right] \subseteq R[F]$. An $R\left[F^{e}\right]$-module is hence nothing but an $R$-module $M$ together with an "action of the $e^{\text {th }}$ composition of the Frobenius on $M$ ", that is, a group homomorphism $F^{e}=F_{M}^{e}: M \rightarrow M$ such that $F^{e}(a u)=a^{p^{e}} u$ (or more concisely: $F_{M}^{e}$ is an $R$-linear map $M \rightarrow F_{*}^{e} M$ ). Due to the adjointness of $F^{e *}$ and $F_{*}^{e}$ this can be rephrased as follows: $M$ is an $R$-module together with a morphism of left $R$ modules $\vartheta_{M}^{e}: R^{e} \otimes_{R} M \rightarrow M$. The adjointness is expressed through the equation $\vartheta_{M}^{e}(a \otimes u)=a F^{e}(u)$.

A unit $R\left[F^{e}\right]$-module is an $R\left[F^{e}\right]$-module $M$ such that $\vartheta_{M}^{e}$ is an isomorphism. Note that for every $s \geq 1$, the inclusion $R\left[F^{s e}\right] \subseteq R\left[F^{e}\right]$ makes any (unit) $R\left[F^{e}\right]$ module into a (unit) $R\left[F^{s e}\right]$-module. Moreover, $\vartheta_{M}^{s e}$ can be described recursively as

$$
R^{s e} \otimes_{R} M \simeq R^{e} \otimes_{R}\left(R^{(s-1) e} \otimes_{R} M\right) \stackrel{1 \vartheta_{M}^{(s-1) e}}{\longrightarrow} R^{e} \otimes_{R} M \stackrel{\vartheta_{M}^{e}}{\longrightarrow} M .
$$

Every unit $R\left[F^{e}\right]$-module $M$ has a canonical structure of a $D_{R}$-module. This is described as follows: since $D_{R}=\bigcup_{s>1} D_{R}^{s e}$, it is enough to describe the action of $P \in D_{R}^{s e}$ on $M$. Using the isomorphism $\vartheta_{M}^{s e}: R^{s e} \otimes_{R} M \rightarrow M$, we let $P$ act by $P(a \otimes u)=P(a) \otimes u$. A fundamental result of Lyubeznik $\mathrm{Lyu}$ says that if $R$ is an algebra of finite type over a regular local $F$-finite ring, then every finitely generated unit $R\left[F^{e}\right]$-module has finite length in the category of $D_{R}$-modules.

It is a general fact that for every $R$-module $P$ and every $e \geq 1$, the pull-back

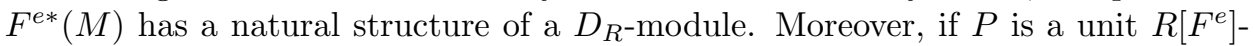

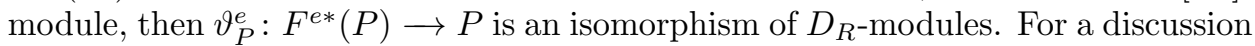
of this and related facts we refer to [AMBL, $\S 2$.

For simplicity, from now on we assume that $R$ is a domain. A basic example of an $R[F]$-module is given by $R_{f}$, where $f \in R$ is nonzero. The action of $F$ on $R_{f}$ is given by the Frobenius morphism of $R_{f}$. It is easy to see that $R_{f}$ is a unit $R[F]$-module. In fact, we will check this for the following generalization.

Suppose that $\alpha$ is a positive rational number such that $p$ does not divide the denominator of $\alpha$. Therefore we can find positive integers $e$ and $r$ such that $\alpha=$ $\frac{r}{p^{e}-1}$.

We define the $R\left[F^{e}\right]$-module $M_{\alpha}$ as being the $R_{f}$-free module with generator $e_{\alpha}$. We think of $e_{\alpha}$ formally as $\frac{1}{f^{\alpha}}$. Since $p^{e} \alpha=r+\alpha$, this suggests the following action of $F^{e}$ on $M_{\alpha}$ :

$$
F^{e}\left(\frac{b}{f^{m}} \cdot e_{\alpha}\right)=\frac{b^{p^{e}}}{f^{m p^{e}+r}} \cdot e_{\alpha} .
$$

It is clear that this makes $M_{\alpha}$ an $R\left[F^{e}\right]$-module.

Lemma 2.3. For every $\alpha$ as above, $M_{\alpha}$ is a unit $R\left[F^{e}\right]$-module.

Proof. It follows from definition that the morphism $\vartheta_{M_{\alpha}}^{e}: R^{e} \otimes_{R} M_{\alpha} \rightarrow M_{\alpha}$ is given by

$$
\vartheta_{M_{\alpha}}^{e}\left(a \otimes \frac{b}{f^{m}} e_{\alpha}\right)=\frac{a b^{p^{e}}}{f^{m p^{e}+r}} e_{\alpha} .
$$

It is straightforward to check that the map $\frac{c}{f^{s}} e_{\alpha} \rightarrow c f^{s\left(p^{e}-1\right)+r} \otimes \frac{1}{f^{s}} e_{\alpha}$ is welldefined and that it is an inverse of $\vartheta_{M_{\alpha}}^{e}$. 
Remark 2.4. If $e^{\prime}=e s$ for some positive integer $s$, then we may write

$$
\alpha=\frac{r}{p^{e}-1}=\frac{r^{\prime}}{p^{e^{\prime}}-1},
$$

with $r^{\prime}=r \cdot \frac{p^{e^{\prime}}-1}{p^{e}-1}$. Since

$$
\left(F^{e}\right)^{s}\left(e_{\alpha}\right)=\frac{1}{f^{r\left(1+p^{e}+\cdots+p^{(s-1) e}\right)}} e_{\alpha}=\frac{1}{f^{r^{\prime}}} e_{\alpha},
$$

we see that the action of $F^{e^{\prime}}$ on $e_{\alpha}$ is the same for both ways of writing $\alpha$ in (1). In particular, the $D_{R}$-module structure on $M_{\alpha}$ depends only on $\alpha$.

The following lemma relates the module $M_{\alpha}$ to some test ideals of $f$. If $\alpha=\frac{r}{p^{e}-1}$ as above and $m \in \mathbb{N}$, we put $\alpha_{m}:=\frac{p^{m e}-1}{p^{m e}} \cdot \alpha$. Hence the $\alpha_{m}$ form a strictly increasing sequence converging to $\alpha$.

Lemma 2.5. With the above notation, the following are equivalent:

(i) $\tau\left(f^{\alpha_{m}}\right)=\tau\left(f^{\alpha_{m+1}}\right)$.

(ii) There is a differential operator $P \in D_{R}^{(m+1) e}$ such that $P \cdot e_{\alpha}=F^{e}\left(e_{\alpha}\right)$.

Proof. It follows from Lemma 2.1 that we have

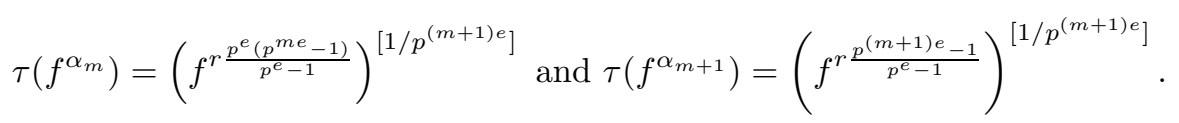

Therefore Lemma 2.2 implies

$\tau\left(f^{\alpha_{m}}\right)^{\left[p^{(m+1) e}\right]}=D_{R}^{(m+1) e} \cdot f^{r \frac{p^{e}\left(p^{m e}-1\right)}{p^{e}-1}}$ and $\tau\left(f^{\alpha_{m+1}}\right)^{\left[p^{(m+1) e}\right]}=D_{R}^{(m+1) e} \cdot f^{r \frac{p^{(m+1) e}-1}{p^{e}-1}}$.

We always have $\tau\left(f^{\alpha_{m+1}}\right) \subseteq \tau\left(f^{\alpha_{m}}\right)$ for every $m$. It follows from the above formulas that these ideals are equal if and only if there is $P \in D_{R}^{(m+1) e}$ such that

$$
f^{\frac{p^{e}\left(p^{m e}-1\right)}{p^{e}-1}}=P \cdot f^{r \frac{p^{(m+1) e}-1}{p^{e}-1}} .
$$

We claim that this is the case if and only if $P \cdot e_{\alpha}=\frac{1}{f^{r}} e_{\alpha}$ in $M_{\alpha}$. Note first that since $P \in D_{R}^{(m+1) e}$, it follows from the description of the action of $D_{R}$ on $M_{\alpha}$ that

$$
P \cdot e_{\alpha}=\vartheta_{M_{\alpha}}^{(m+1) e}(P \otimes 1)\left(\vartheta_{M_{\alpha}}^{(m+1) e}\right)^{-1}\left(e_{\alpha}\right) .
$$

The formula for $\left(\vartheta_{M_{\alpha}}^{e}\right)^{-1}$ in the proof of Lemma 2.3 implies that

$$
\left(\vartheta_{M_{\alpha}}^{(m+1) e}\right)^{-1}\left(e_{\alpha}\right)=f^{r \frac{p^{(m+1) e}-1}{p^{e}-1}} \otimes e_{\alpha},
$$

and therefore

$$
\left(\vartheta_{M_{\alpha}}^{(m+1) e}\right)^{-1}\left(P \cdot e_{\alpha}\right)=P\left(f^{r \frac{p^{(m+1) e}-1}{p^{e}-1}}\right) \otimes e_{\alpha}=f^{r p^{(m+1) e}} \cdot P\left(f^{r \frac{p^{(m+1) e}-1}{p^{e}-1}}\right) \otimes \frac{1}{f^{r}} e_{\alpha} .
$$

On the other hand,

$$
\left(\vartheta_{M_{\alpha}}^{(m+1) e}\right)^{-1}\left(\frac{1}{f^{r}} e_{\alpha}\right)=f^{r \frac{p^{e}\left(p^{(m+1) e}-1\right)}{p^{e}-1}} \otimes \frac{1}{f^{r}} e_{\alpha} ;
$$

hence $P \cdot e_{\alpha}=\frac{1}{f^{r}} e_{\alpha}$ if and only if

$$
f^{r p^{(m+1) e}} P\left(f^{r \frac{p^{(m+1) e}-1}{p^{e}-1}}\right)=f^{r \frac{p^{e}\left(p^{(m+1) e}-1\right)}{p^{e}-1}},
$$

which is equivalent to (2). This completes the proof of the lemma. 
Remark 2.6. Since we have $D_{R}^{(m+1) e} \subseteq D_{R}^{(m+2) e}$, it follows from the lemma that if $\tau\left(f^{\alpha_{m}}\right)=\tau\left(f^{\alpha_{m+1}}\right)$, then $\tau\left(f^{\alpha_{i}}\right)=\tau\left(f^{\alpha_{m}}\right)$ for every $i \geq m$. In other words, there is no $F$-jumping exponent in $\left(\alpha_{m}, \alpha\right)$. See also Proposition 4.3 below for an alternative proof of this statement.

Remark 2.7. Using the language of roots and generators of finitely generated unit $R\left[F^{e}\right]$-modules as in $\overline{\mathrm{Lyu}}$, one can easily show that $M_{\alpha}$ is naturally isomorphic to the unit $R\left[F^{e}\right]$-module generated by

$$
\beta: R \stackrel{a \mapsto f^{r} \cdot a \otimes 1}{\longrightarrow} R^{e} \otimes R \cong F^{e *} R .
$$

The unit module generated by $\beta$ is by definition the inductive limit $\widetilde{M}_{\alpha}$ of the direct system one obtains by composition of Frobenius powers of the map $\beta$. As an $R$-module, $\widetilde{M}_{\alpha}$ is isomorphic to $R_{f}$, but the action of the Frobenius is not the usual one (except in the case $r=p^{e}-1$ ). One easily checks (by sending the image of $1 \in R$ in the limit $\widetilde{M}_{\alpha}$ to $e_{\alpha} \in M_{\alpha}$ ) that $\widetilde{M}_{\alpha}$ and $M_{\alpha}$ are isomorphic as $R\left[F^{e}\right]$ modules. By construction it follows that $R e_{\alpha} \subseteq M_{\alpha}$ is a root of $M_{\alpha}$. Therefore AMBL, Corollary 4.4] implies that $e_{\alpha}$ generates $M_{\alpha}$ as a $D_{R}$-module. In Theorem 2.11 below we will give a direct proof of this fact.

Corollary 2.8. If $\alpha=\frac{r}{p^{e}-1}$, then $e_{\alpha}$ generates $M_{\alpha}$ as a $D_{R}$-module if and only if $\alpha$ is not an accumulation point of F-jumping exponents of $f$.

Proof. The $\alpha_{m}$ form a strictly increasing sequence converging to $\alpha$. Hence $\alpha$ is not an accumulation point of $F$-jumping exponents if and only if the sequence of ideals $\left\{\tau\left(f^{\alpha_{m}}\right)\right\}_{m}$ stabilizes. By Remark 2.6. this is the case if and only if $\tau\left(f^{\alpha_{m}}\right)=\tau\left(f^{\alpha_{m+1}}\right)$ for some $m$. Since $D_{R}=\bigcup_{m>1} D_{R}^{m e}$, it is clear from Lemma2.5 that if $M_{\alpha}=D_{R} \cdot e_{\alpha}$, then $\alpha$ is not an accumulation point of $F$-jumping exponents. Conversely, if this is the case, then $\frac{1}{f^{r}} e_{\alpha} \in D_{R} \cdot e_{\alpha}$. By Remark 2.4, we see that in fact we have infinitely many positive integers $r_{m}$ such that $\frac{1}{f^{r_{m}}} e_{\alpha}$ lies in $D_{R} \cdot e_{\alpha}$. Since these elements generate $M_{\alpha}$ as an $R$-module, we see that $M_{\alpha}=D_{R} \cdot e_{\alpha}$.

Corollary 2.9. If $\alpha=\frac{r}{p^{e}-1}$, then $\alpha$ is not an accumulation point of $F$-jumping exponents of $f \in R$ if and only if for every $\mathfrak{q} \in \operatorname{Spec}(R), \alpha$ is not an accumulation point of F-jumping exponents of $\frac{f}{1} \in R_{\mathfrak{q}}$.

Proof. We have seen that $\alpha$ is not an accumulation point of $F$-jumping exponents of $f$ if and only if $\tau\left(f^{\alpha_{m}}\right)=\tau\left(f^{\alpha_{m+1}}\right)$ for some $m$. Since taking test ideals commutes with localization, it is clear that if this property holds in $R$, then it holds in every $R_{\mathfrak{q}}$. For the converse, note that if

$$
\tau\left(\left(f R_{\mathfrak{q}}\right)^{\alpha_{m}}\right)=\tau\left(\left(f R_{\mathfrak{q}}\right)^{\alpha_{m+1}}\right),
$$

then the same holds for all primes $\mathfrak{q}^{\prime}$ in a neighborhood of $\mathfrak{q}$. If $U_{m}$ is the open subset consisting of those $\mathfrak{q}$ for which (3) holds and if $\operatorname{Spec}(R)=\bigcup_{m} U_{m}$, then $\operatorname{Spec}(R)=U_{m_{0}}$ for some $m_{0}$ (we use the fact that $\operatorname{Spec}(R)$ is quasicompact and that $U_{m} \subseteq U_{m+1}$ by Remark 2.6). This implies that $\tau\left(f^{\alpha_{m_{0}}}\right)=\tau\left(f^{\alpha_{m_{0}+1}}\right)$, hence $\alpha$ is not an accumulation point of $F$-exponents of $f$.

Remark 2.10. It is easy to see that $M_{\alpha}$ is generated by $e_{\alpha}$ as an $R\left[F^{e}\right]$-module. Indeed, $M_{\alpha}$ is generated as an $R$-module by the $\left(F^{e}\right)^{m}\left(e_{\alpha}\right)=\frac{1}{f^{r \frac{p^{m e}-1}{p^{e}-1}}} \cdot e_{\alpha}$, with $m \geq 1$. 
Theorem 2.11. Let $R$ be an $F$-finite regular domain. If $f$ is a nonzero element in $R$ and $\alpha=\frac{r}{p^{e}-1}$ for some positive integers $r$ and $e$, then $M_{\alpha}$ is generated over $D_{R}$ by $e_{\alpha}$.

Proof. Note first that by Corollary 2.9, we may assume that $R$ is local. Then the argument follows verbatim the argument for Theorem 4.1 in [AMBL]. Let $N$ denote the $D_{R}$-submodule of $M_{\alpha}$ generated by $e_{\alpha}$. Note that we have

$$
F^{e *}(N) \subseteq F^{e *}\left(M_{\alpha}\right) \stackrel{\vartheta_{M_{\alpha}}^{e}}{\longrightarrow} M_{\alpha} ;
$$

hence we may consider $F^{e *}(N)$ as a submodule of $M_{\alpha}$. We claim that $N \subseteq F^{e *}(N)$.

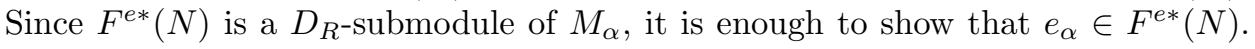
This follows from $e_{\alpha}=f^{r} \cdot \vartheta_{M_{\alpha}}^{e}\left(1 \otimes e_{\alpha}\right)$.

Theorem 4.3 in AMBL shows that this makes $N$ a unit $R\left[F^{e}\right]$-module, i.e., we have in fact $N=F^{* *}(N)$. The idea is the following: if $N \neq\left(F^{*}\right)(N)$, then the faithful flatness of the Frobenius implies that we have a sequence of strict inclusions

$$
N \subsetneq F^{e *}(N) \subsetneq\left(F^{2 e}\right)^{*}(N) \subsetneq \cdots
$$

of $D_{R}$-submodules of $M_{\alpha}$. This contradicts Lyubeznik's Theorem Lyu which says that as a unit $R\left[F^{e}\right]$-module, $M_{\alpha}$ has finite length in the category of $D_{R}$-modules (we may apply the theorem, since we assume that $R$ is local and $F$-finite).

Therefore we have $N=\left(F^{m e}\right)^{*}(N)$ for every $m$. On the other hand, every element in $M_{\alpha}$ lies in some $\left(F^{m e}\right)^{*}(N)$. This follows from

$$
\frac{1}{f^{r p^{(m-1) e}}} e_{\alpha}=\vartheta_{M_{\alpha}}^{m e}\left(1 \otimes e_{\alpha}\right) \in\left(F^{m e}\right)^{*}(N)
$$

Therefore $N=M_{\alpha}$.

Remark 2.12. By putting together the above results, we see that under the hypothesis of Theorem [2.11, every rational number $\alpha$ whose denominator is not divisible by $p$ is not an accumulation point of $F$-jumping exponents of a given $f$. The above proofs extend to this setting the main result in AMBL, which deals with the case $\alpha=1$. In addition, we have dropped the extra assumption that was imposed in loc. cit. in order to apply Lyubeznik's Theorem.

Before we proceed to the proof of Theorem 1.1 we show the following lemma which allows us to do induction. Note that this lemma itself does not require the ideal to be principal.

Lemma 2.13. Let $R$ be an $F$-finite regular ring, and $\mathfrak{a}$ an ideal in $R$.

(i) If $\lambda$ is an $F$-jumping exponent of $\mathfrak{a}$, then there is a prime ideal $\mathfrak{q}$ in $R$ such that $\lambda$ is also an $F$-jumping exponent of $\mathfrak{a} R_{\mathfrak{q}}$.

(ii) If $\lambda$ is an accumulation point of jumping numbers of $\mathfrak{a}$, then we can find a nonmaximal prime ideal $\mathfrak{q}$ such that $\lambda$ is an F-jumping exponent of $\mathfrak{a} R_{\mathfrak{q}}$.

Proof. We may assume that $\lambda>0$, and let us fix a strictly increasing sequence of positive numbers $\left\{\lambda_{m}\right\}_{m}$, with $\lim _{m \rightarrow \infty} \lambda_{m}=\lambda$. For every $m$ we have $\tau\left(\mathfrak{a}^{\lambda}\right) \subseteq$ $\tau\left(\mathfrak{a}^{\lambda_{m+1}}\right) \subseteq \tau\left(\mathfrak{a}^{\lambda_{m}}\right)$. Let $I_{m}$ be the ideal

$$
\left(\tau\left(\mathfrak{a}^{\lambda}\right): \tau\left(\mathfrak{a}^{\lambda_{m}}\right)\right)=\left\{h \in R \mid h \cdot \tau\left(\mathfrak{a}^{\lambda_{m}}\right) \subseteq \tau\left(\mathfrak{a}^{\lambda}\right)\right\} .
$$

Therefore $I_{m} \subseteq I_{m+1}$ for every $m$, and since $R$ is Noetherian, there is an ideal $I$ such that $I_{m}=I$ for all $m \gg 0$. 
Note that $\lambda$ is an $F$-jumping exponent of $\mathfrak{a}$ if and only if for every $m$ we have $\tau\left(\mathfrak{a}^{\lambda}\right) \neq \tau\left(\mathfrak{a}^{\lambda_{m}}\right)$, or equivalently, $I_{m} \neq R$. Moreover, $\lambda$ is an accumulation point of $F$-jumping exponents if and only if $\tau\left(\mathfrak{a}^{\lambda_{m}}\right) \neq \tau\left(\mathfrak{a}^{\lambda_{m+1}}\right)$ for every $m$.

If $\lambda$ is an $F$-jumping exponent of $\mathfrak{a}$, let $\mathfrak{q}$ be a minimal prime containing $I$. Since

$$
\left(\tau\left(\left(\mathfrak{a} R_{\mathfrak{q}}\right)^{\lambda}\right): \tau\left(\left(\mathfrak{a} R_{\mathfrak{q}}\right)^{\lambda_{m}}\right)\right)=\left(\tau\left(\mathfrak{a}^{\lambda}\right) R_{\mathfrak{q}}: \tau\left(\mathfrak{a}^{\lambda_{m}}\right) R_{\mathfrak{q}}\right)=I_{\mathfrak{q}} \neq R_{\mathfrak{q}},
$$

it follows that $\lambda$ is an $F$-jumping exponent of $\mathfrak{a} R_{\mathfrak{q}}$. This gives (i).

We now show that if $\lambda$ is an accumulation point of $F$-jumping exponents, then we can find $\mathfrak{q}$ as above that is not a maximal ideal. Equivalently, we need to show that $\operatorname{dim}(R / I) \geq 1$, i.e., $R / I$ is not Artinian. By assumption, if $m \gg 0$, then we have a strictly decreasing sequence

$$
\tau\left(\mathfrak{a}^{\lambda_{m}}\right) / \tau\left(\mathfrak{a}^{\lambda}\right) \supsetneq \tau\left(\mathfrak{a}^{\lambda_{m+1}}\right) / \tau\left(\mathfrak{a}^{\lambda}\right) \supsetneq \ldots
$$

of finitely generated $R / I$-modules. Therefore $R / I$ cannot be Artinian, and we get (ii).

Proof of Theorem 1.1. Since $R$ is a regular ring, we may write $R=R_{1} \times \cdots \times R_{m}$, where $R_{i}$ are regular domains. If we write $f=\left(f_{1}, \ldots, f_{m}\right)$, then the set of $F$ jumping exponents of $f$ is the union of the sets of $F$-jumping exponents of each of the $f_{i}$. Therefore, in order to prove Theorem 1.1 for $R$, we may assume that $R$ is a domain and that $f \neq 0$, the case $f=0$ being trivial.

We have seen that for every $R$ and $f$, the discreteness of the set of $F$-jumping exponents implies the rationality of every such exponent. Conversely, if we know that all $F$-jumping exponents are rational, then they form a discrete set. Indeed, if $\alpha$ is an accumulation point of $F$-jumping exponents, then $\alpha$ is an $F$-jumping exponent, too; hence $\alpha \in \mathbb{Q}$. We can find a positive integer $m$ such that the denominator of $p^{m} \alpha$ is not divisible by $p$. For every $F$-jumping exponent $\beta, p^{m} \beta$ is again an $F$-jumping exponent. Therefore also $p^{m} \alpha$ is an accumulation point of $F$-jumping exponents, contradicting Theorem 2.11 (see also Remark 2.12).

We claim that it is enough to prove Theorem 1.1 when $R$ is local. Indeed, given an arbitrary regular $F$-finite local ring, if $\alpha$ is an $F$-jumping exponent of $f$, then $\alpha$ is also an $F$-jumping exponent for $f R_{\mathfrak{q}}$ for some prime $\mathfrak{q}$, by Lemma 2.13 , Hence $\alpha \in \mathbb{Q}$, by the local case, and, as we have seen, this implies that $R$ satisfies the theorem.

Suppose now that $R$ is local. In particular, $\operatorname{dim}(R)<\infty$, and we prove the statement by induction on $\operatorname{dim}(R)$. The case $\operatorname{dim}(R)=0$ is trivial; hence we may assume that the theorem holds for local rings of dimension $<\operatorname{dim}(R)$. If $\alpha$ is an accumulation point of $F$-jumping exponents, then Lemma 2.13 implies that there is a prime ideal $\mathfrak{q}$ in $R$, different from the maximal ideal, such that $\alpha$ is an $F$-jumping exponent for $f R_{\mathfrak{q}}$. Since $\operatorname{dim}\left(R_{\mathfrak{q}}\right)<\operatorname{dim}(R)$, we may apply induction to conclude that $\alpha \in \mathbb{Q}$. Arguing as before, we get a contradiction with Theorem 2.11. This implies that the set of $F$-jumping numbers of $f$ is discrete, and as a consequence, it contains only rational numbers.

\section{Limits OF $F$-PURE THRESHOLDS}

The $F$-pure threshold of an ideal has been introduced in [TW] and further studied in MTW. We start by recalling some basic properties. In fact, it is convenient to consider more generally the interpretation of all $F$-jumping exponents of an ideal as $F$-thresholds as follows. We refer to BMS for details and proofs. 
Suppose that $R$ is an $F$-finite regular ring, $\mathfrak{a}$ is an ideal in $R$, and $J$ is an ideal such that $\mathfrak{a} \subseteq \operatorname{Rad}(J)$. For every $e$ let $\nu\left(p^{e}\right)$ denote the largest $r$ such that $\mathfrak{a}^{r} \nsubseteq J^{\left[p^{e}\right]}$ (if there is no such $r$, we put $\nu\left(p^{e}\right)=0$ ). By the flatness of the Frobenius one has that

$$
\nu\left(p^{e}\right) / p^{e} \leq \nu\left(p^{e+1}\right) / p^{e+1}
$$

for every $e$. The limit

$$
c^{J}(\mathfrak{a})=\lim _{e \rightarrow \infty} \frac{\nu\left(p^{e}\right)}{p^{e}}=\sup _{e \geq 1} \frac{\nu\left(p^{e}\right)}{p^{e}}
$$

is finite, and it is called the $F$-threshold of $\mathfrak{a}$ with respect to $J$. It was shown in BMS, Corollary 2.24, that the set of all $F$-thresholds of $\mathfrak{a}$ (when we vary $J$ ) is equal to the set of $F$-jumping exponents of $\mathfrak{a}$. More precisely, $c^{J}(\mathfrak{a})$ is the smallest $\lambda$ such that $\tau\left(\mathfrak{a}^{\lambda}\right) \subseteq J$. We mention that one can show that for every $e$ we have a strict inequality $\nu\left(p^{e}\right) / p^{e}<c^{J}(\mathfrak{a})$ (see Proposition 1.7 in [MTW]).

Note that $\tau\left(\left(\mathfrak{a}^{m}\right)^{\lambda}\right)=\tau\left(\mathfrak{a}^{m \lambda}\right)$ for every $\lambda$; hence $c^{J}\left(\mathfrak{a}^{m}\right)=c^{J}(\mathfrak{a}) / m$ for every $J$. On the other hand, we have $c^{J^{[p]}}(\mathfrak{a})=p \cdot c^{J}(\mathfrak{a})$.

Remark 3.1. When $\mathfrak{a}=(f)$ is principal, then $f^{r} \in J^{\left[p^{e}\right]}$ implies $f^{p r} \in J^{\left[p^{e+1}\right]}$. Therefore we have

$$
\frac{\nu\left(p^{e+1}\right)+1}{p^{e+1}} \leq \frac{\nu\left(p^{e}\right)+1}{p^{e}} ;
$$

hence $c^{J}(\mathfrak{a})=\inf _{e} \frac{\nu\left(p^{e}\right)+1}{p^{e}}$. It follows that for every ideal $J$ and every $e$, we have $\nu\left(p^{e}\right)+1=\left\lceil c^{J}(f) p^{e}\right\rceil^{e}$

Suppose now that $R$ is a domain. If $(0) \neq \mathfrak{a} \neq R$, then $\tau\left(\mathfrak{a}^{0}\right)=R$, and $\tau\left(\mathfrak{a}^{\lambda}\right) \neq R$ for $\lambda \gg 0$ (in fact, the test ideal is contained in $\mathfrak{a}$ for $\lambda \gg 0$; see, for example, Proposition 2.20 in $[\mathrm{BMS}]$ ). The $F$-pure threshold $\mathrm{fpt}(\mathfrak{a})$ is defined as the smallest positive $F$-jumping exponent of $\mathfrak{a}$, i.e., it is the smallest $\lambda$ such that $\tau\left(\mathfrak{a}^{\lambda}\right) \neq R$. We make the convention that $\operatorname{fpt}(0)=0$ and $\operatorname{fpt}(R)=\infty$.

If $R=R_{1} \times \cdots \times R_{m}$ and $\mathfrak{a}=\mathfrak{a}_{1} \times \cdots \times \mathfrak{a}_{m}$, then we define the $F$-pure threshold of $\mathfrak{a}$ by $\operatorname{fpt}(\mathfrak{a})=\min _{i} \operatorname{fpt}\left(\mathfrak{a}_{i}\right)$. We can use this to reduce the computation of $F$-pure thresholds to the case when $R$ is a domain, which we will do henceforth.

It is clear from the definition that if $\mathfrak{a} \subseteq \mathfrak{b}$, then $\operatorname{fpt}(\mathfrak{a}) \leq \operatorname{fpt}(\mathfrak{b})$. Note also that $\operatorname{fpt}\left(\mathfrak{a}^{m}\right)=\operatorname{fpt}(\mathfrak{a}) / m$. We record in the following proposition a few other useful properties of $F$-pure thresholds.

Proposition 3.2. Let $\mathfrak{a}$ be an ideal in $R$.

(i) If $(R, \mathfrak{m})$ is local and $\mathfrak{a} \neq R$, then $\operatorname{fpt}(\mathfrak{a})=c^{\mathfrak{m}}(\mathfrak{a})$.

(ii) If $S$ is a multiplicative system in $R$, then $\operatorname{fpt}\left(S^{-1} \mathfrak{a}\right) \geq \operatorname{fpt}(\mathfrak{a})$.

(iii) If $\mathfrak{m}$ is a maximal ideal containing $\mathfrak{a}$, then $\operatorname{fpt}\left(\mathfrak{a} R_{\mathfrak{m}}\right)=c^{\mathfrak{m}}(\mathfrak{a})$.

(iv) We have $\operatorname{fpt}(\mathfrak{a})=\min _{\mathfrak{q}} \operatorname{fpt}\left(\mathfrak{a} R_{\mathfrak{q}}\right)$, where the minimum is either over the prime ideals or over the maximal ideals of $R$.

(v) If $R$ is a local ring and $\widehat{R}$ is its completion, then $\operatorname{fpt}(\mathfrak{a} \widehat{R})=\operatorname{fpt}(\mathfrak{a})$.

Proof. For all assertions we may assume that $R$ is a domain and that $\mathfrak{a}$ is a proper nonzero ideal. (i) is clear, and (ii) follows from the fact that taking test ideals commutes with localization. For (iii), note that since $\mathfrak{m}^{\left[p^{e}\right]}$ is $\mathfrak{m}$-primary, we get $\left(\mathfrak{m} R_{\mathfrak{m}}\right)^{\left[p^{e}\right]} \cap R=\mathfrak{m}^{\left[p^{e}\right]}$, which gives our statement. 
In order to prove (iv), let $c=\operatorname{fpt}(\mathfrak{a})$. If $I=\tau\left(\mathfrak{a}^{c}\right)$, then for every prime ideal $\mathfrak{q}$ containing $I$ we have $\tau\left(\left(\mathfrak{a} R_{\mathfrak{q}}\right)^{c}\right)=I_{\mathfrak{q}} \neq R_{\mathfrak{q}}$; hence $c=\operatorname{fpt}\left(\mathfrak{a} R_{\mathfrak{q}}\right)$. (v) follows from the fact that taking test ideals commutes with completion.

The following lemma will allow us to approximate arbitrary $F$-pure thresholds by $F$-pure thresholds of polynomials. The statement can be found in [MTW], but we give the proof for completeness.

Lemma 3.3. If $J$ is an ideal in $R$ and $\mathfrak{a}, \mathfrak{b}$ are ideals contained in $\operatorname{Rad}(J)$, then $c^{J}(\mathfrak{a}+\mathfrak{b}) \leq c^{J}(\mathfrak{a})+c^{J}(\mathfrak{b})$.

Proof. If $e \geq 1$ and $r, s$ are such that $\mathfrak{a}^{r} \subseteq J^{\left[p^{e}\right]}$ and $\mathfrak{b}^{s} \subseteq J^{\left[p^{e}\right]}$, then it is clear that $(\mathfrak{a}+\mathfrak{b})^{r+s} \subseteq J^{\left[p^{e}\right]}$. The assertion of the lemma now follows from the definition of $F$-thresholds.

Corollary 3.4. If $\mathfrak{m}^{\left[p^{s}\right]} \subseteq J \subseteq \mathfrak{m}$ for some maximal ideal $\mathfrak{m}$ and some $s \geq 1$, and if $f, g \in \mathfrak{m}$ are such that $f-g \in \mathfrak{m}^{N}$, then

$$
\left|c^{J}(f)-c^{J}(g)\right| \leq \frac{p^{s} \cdot \operatorname{dim}\left(R_{\mathfrak{m}}\right)}{N} .
$$

Proof. Since $f-g \in \mathfrak{m}^{N}$, the lemma gives

$$
\left|c^{J}(f)-c^{J}(g)\right| \leq c^{J}\left(\mathfrak{m}^{N}\right) \leq c^{\mathfrak{m}^{\left[p^{s}\right]}}\left(\mathfrak{m}^{N}\right)=\frac{p^{s} \cdot \operatorname{dim}\left(R_{\mathfrak{m}}\right)}{N},
$$

where we use the fact that $c^{\mathfrak{m}}(\mathfrak{m})=\operatorname{dim}\left(R_{\mathfrak{m}}\right)$.

We now turn to the study of the set of $F$-pure thresholds of principal ideals in bounded dimension. Recall that since $\tau(f)=(f)$, we have $\operatorname{fpt}(f) \leq 1$ for every noninvertible $f$ in a domain $R$. If $k$ is a field and $f \in k\left[x_{1}, \ldots, x_{n}\right]$ is such that $f(0)=0$, then we denote by $\operatorname{fpt}_{0}(f)$ the $F$-pure threshold of the image of $f$ in $k\left[x_{1}, \ldots, x_{n}\right]_{\left(x_{1}, \ldots, x_{n}\right)}$.

We now fix the characteristic $p$. For $n \geq 1$ and for every field $k$ of characteristic $p$ we denote by $\mathcal{T}_{n}^{\circ}(k)$ the set of $F$-pure thresholds fpt $f_{0}(f)$, where $f \in k\left[x_{1}, \ldots, x_{n}\right]$ is such that $f(0)=0$. We also put $\mathcal{T}_{n}$ for the set of $F$-pure thresholds $\operatorname{fpt}(g)$, where $g \in R$ is not invertible and $R$ is an $F$-finite regular domain of dimension $\leq n$.

Theorem 3.5. If the characteristic $p$ is fixed, then

(i) For every $n$ we have $\mathcal{T}_{n}^{\circ}(k) \subseteq \mathcal{T}_{n+1}^{\circ}(k)$.

(ii) For every field extension $K / k$, we have $\mathcal{T}_{n}^{\circ}(k) \subseteq \mathcal{T}_{n}^{\circ}(K)$.

(iii) The set $\mathcal{T}_{n}^{\circ}(k)$ does not depend on $k$ if $k$ is algebraically closed (from now on we simply denote this set by $\left.\mathcal{T}_{n}^{\circ}\right)$.

(iv) The set $\mathcal{T}_{n}$ is contained in the closure of $\mathcal{T}_{n}^{\circ}$.

Proof. If $f \in k\left[x_{1}, \ldots, x_{n}\right]$, then $\operatorname{fpt}_{0}(f)=\lim _{e \rightarrow \infty} \nu\left(p^{e}\right) / p^{e}$, where $\nu\left(p^{e}\right)$ is the largest $r$ such that $f^{r} \notin\left(x_{1}^{p^{e}}, \ldots, x_{n}^{p^{e}}\right)$. It is clear that $\nu\left(p^{e}\right)$ does not change if we consider instead $f$ in $k\left[x_{1}, \ldots, x_{n}\right]$ or in $K\left[x_{1}, \ldots, x_{n}\right]$, for some field extension $K$ of $k$. This gives the first two assertions.

For (iii), it is enough to show that for every $d \geq 1$ and $c>0$, the existence of $f \in k\left[x_{1}, \ldots, x_{n}\right]_{\leq d}$ (these are the polynomials of degree $\leq d$ ) with $\mathrm{fpt}_{0}(f)=c$ does not depend on the algebraically closed field $k$. Note that

$$
\left\{f \in k\left[x_{1}, \ldots, x_{n}\right]_{\leq d} \mid f(0)=0\right\} \cong k^{N}
$$


can be viewed as the set of $k$-valued points of a suitable affine space $\mathbb{A}^{N}$. Recall first from BMS, Proposition 3.6] that the denominators of the $F$-jumping numbers of $f$ in the above set are bounded in terms of $d, n$ and the characteristic $p$; in particular, the bound is independent of the field $k$. Therefore we have $0<a_{1}<$ $\cdots<a_{m(d, n, p)} \leq 1$ such that for every nonzero $f$ of degree $\leq d$ and with $f(0)=0$, we have $\mathrm{fpt}_{0}(f)=a_{i}$ for some $i$. From now on we may assume that $c=a_{j}$ for some $j$.

On the other hand, for every $a>0$, the condition that $\mathrm{fpt}_{0}(f) \leq a$ is equivalent with $f^{\left\lfloor a p^{e}\right\rfloor+1} \in\left(x_{1}^{p^{e}}, \ldots, x_{n}^{p^{e}}\right)$ for every $e$. Here we have denoted by $\lfloor u\rfloor$ the largest integer $\leq u$. Since the degree of $f$ is bounded above by $d$, it follows that the condition for $f$ to have $\operatorname{fpt}_{0}(f) \geq a$ is an intersection of closed conditions defined over $\mathbb{F}_{p}$; hence it is closed and defined over $\mathbb{F}_{p}$. In other words, there is a closed subscheme $Z_{a} \subseteq \mathbb{A}_{\mathbb{F}_{p}}^{N}$, such that $f \in k\left[x_{1}, \ldots, x_{n}\right]_{\leq d}$ with $f(0)=0 \operatorname{has}_{\operatorname{fpt}_{0}}(f) \leq a$ if and only if $f$ corresponds to a $k$-valued point of $Z_{a}$.

Given $c=a_{j}$, let us choose $a$ and $b$ such that $a_{j-1}<a<a_{j}<b<a_{j+1}$. It follows that there is $f \in k\left[x_{1}, \ldots, x_{n}\right]_{\leq d}$ with $\mathrm{fpt}_{0}(f)=a_{j}$ if and only if $Z_{a}(k) \neq$ $Z_{b}(k)$. This condition does not depend on $k$ if $k$ is algebraically closed, which proves (iii).

In order to prove (iv), consider $c \in \mathcal{T}_{n}$. By definition, there is an $F$-finite regular ring $R$ of dimension $\leq n$ and $g \in R$ noninvertible such that $c=\operatorname{fpt}(g)$. By (iv) and (v) in Proposition 3.2. we may assume that $R$ is local and complete. Since $R$ is regular and contains a field, Cohen's Structure Theorem implies that $R \simeq$ $k \llbracket x_{1}, \ldots, x_{m} \rrbracket$ for some field $k$ and some $m \leq n$. Since $k$ is a quotient of $R$, it follows that $\left[k: k^{p}\right]<\infty$. Using Proposition 3.2 again we see that if $f \in k\left[x_{1}, \ldots, x_{m}\right]$ with $f(0)$, then $\mathrm{fpt}_{0}(f)$ is equal to its $F$-pure threshold in $R$. If we denote by $g_{\leq d}$ the truncation of $g$ up to degree $\leq d$, it follows from Corollary 3.4 that

$$
\left|\operatorname{fpt}_{0}\left(g_{\leq d}\right)-\operatorname{fpt}(g)\right| \leq \frac{m}{d+1} .
$$

This implies that $c=\operatorname{fpt}(g)$ is in the closure of $\mathcal{T}_{n}^{\circ}$.

Our next goal is to prove Theorem [1.2. In order to show that $\mathcal{T}_{n}$ is closed, the key idea is to associate to a sequence of polynomials $f_{m} \in k\left[x_{1}, \ldots, x_{n}\right]$ with $\lim _{m \rightarrow \infty} \operatorname{fpt}_{0}\left(f_{m}\right)=\alpha$ a formal power series $f$ over some extension field of $k$ such that $\operatorname{fpt}(f)=\alpha$. This will be done using some basic constructions from nonstandard analysis. We briefly present these constructions and refer to Gol for details.

Recall that an ultrafilter $\mathcal{U}$ on the set $\mathbb{N}$ is a collection of subsets of $\mathbb{N}$ with the following properties:

(i) $\emptyset \notin \mathcal{U}$.

(ii) If $A \in \mathcal{U}$ and $B \supseteq A$, then $B \in \mathcal{U}$.

(iii) If $A, B \in \mathcal{U}$, then $A \cap B \in \mathcal{U}$.

(iv) If $A \subseteq \mathbb{N}$, then either $A$ or $\mathbb{N} \backslash A$ is in $\mathcal{U}$.

An ultrafilter $\mathcal{U}$ is nonprincipal if every subset of $\mathbb{N}$ whose complement is finite belongs to $\mathcal{U}$. It follows from Zorn's Lemma that there are nonprincipal ultrafilters on $\mathbb{N}$, and from now on we fix one such nonprincipal ultrafilter $\mathcal{U}$. Since we will need this later we point out that the properties (iii) and (iv) easily imply that if $A=U_{1} \cup \cdots \cup U_{n}$ is a finite disjoint union, and if $A \in \mathcal{U}$, then one and only one of the $U_{i}$ is in $\mathcal{U}$. 
If $\left\{A_{m}\right\}_{m \in \mathbb{N}}$ is a sequence of sets, then one defines on the product $\prod_{m \in \mathbb{N}} A_{m}$ the equivalence relation

$$
\left(a_{m}\right)_{m} \sim\left(b_{m}\right)_{m} \text { iff }\left\{m \mid a_{m}=b_{m}\right\} \in \mathcal{U}
$$

The set of equivalence classes is called the ultraproduct (with respect to the ultrafilter $\mathcal{U})$, and we denote it here by $\left[A_{m}\right]$; the class of $\left(a_{m}\right)_{m}$ is denoted by $\left[a_{m}\right]$. Similarly, from a sequence of functions $f_{m}: A_{m} \rightarrow B_{m}$ we get a function $\left[f_{m}\right]:\left[A_{m}\right] \rightarrow\left[B_{m}\right]$ that takes $\left[a_{m}\right]$ to $\left[f\left(a_{m}\right)\right]$.

When $A_{m}=A$ for every $m$, the corresponding ultraproduct is denoted by ${ }^{*} A$ and is called the nonstandard extension of $A$. Note that we have an injective map $A \hookrightarrow{ }^{*} A$ that takes $a$ to the class of $(a, a, \ldots)$. Similarly, a function $u: A \longrightarrow B$ has a nonstandard extension ${ }^{*} u:{ }^{*} A \longrightarrow{ }^{*} B$. As a general principle one observes that if $A$ has an algebraic structure, then ${ }^{*} A$ has a similar structure, too. For example, ${ }^{*} \mathbb{R}$ is an ordered field, and if $k$ is an algebraically closed field, then so is ${ }^{*} k$.

If we have a sequence of polynomials $f_{m} \in k\left[x_{1}, \ldots, x_{n}\right]$, then we obtain an internal hyperpolynomial $F=\left[f_{m}\right] \in^{*}\left(k\left[x_{1}, \ldots, x_{n}\right]\right)$. We can view any polynomial $g \in k\left[x_{1}, \ldots, x_{n}\right]$ (or more generally any power series) as a function $\mathbb{N}^{n} \rightarrow k$ given by sending the tuple $\left(m_{1}, \ldots, m_{n}\right)$ to the coefficient of the monomial $x_{1}^{m_{1}} \cdot \ldots \cdot x_{n}^{m_{n}}$ in $g$. Hence we can view $F$ as a function $\left({ }^{*} \mathbb{N}\right)^{n} \rightarrow{ }^{*} k$. If we restrict this function $F$ to $\mathbb{N}^{n}$, then we get a formal power series $f \in\left({ }^{*} k\right) \llbracket x_{1}, \ldots, x_{n} \rrbracket$. Hence we have the following natural maps:

$$
k\left[x_{1}, \ldots, x_{n}\right] \hookrightarrow{ }^{*}\left(k\left[x_{1}, \ldots, x_{n}\right]\right) \rightarrow\left({ }^{*} k\right) \llbracket x_{1}, \ldots, x_{n} \rrbracket .
$$

Note that if $f_{m}(0)=0$ for every $m$, then $f$ lies in the maximal ideal, i.e. $f(0)=0$. After these preparations, we can prove that $\mathcal{T}_{n}$ is closed.

Proof of Theorem 1.2. We fix an algebraically closed field $k$ of characteristic $p$. We have already seen in Theorem 3.5 that $\mathcal{T}_{n}^{\circ}=\mathcal{T}_{n}^{\circ}(k)$ is independent on the choice of $k$, and that it is dense in $\mathcal{T}_{n}$. Therefore, in order to show that $\mathcal{T}_{n}$ is closed, it is enough to show that if we have a sequence $f_{m} \in k\left[x_{1}, \ldots, x_{n}\right]$ with $f_{m}(0)=0$ and $\lim _{m \rightarrow \infty} \operatorname{fpt}_{0}\left(f_{m}\right)=\alpha$, then $\alpha \in \mathcal{T}_{n}$. In fact, we will show that if $f \in\left({ }^{*} k\right) \llbracket x_{1}, \ldots, x_{n} \rrbracket$ is the formal power series associated to $F=\left[f_{m}\right]$ as above, then $\alpha=\operatorname{fpt}(f)$. The function $\mathrm{fpt}_{0}:\left\{g \in k\left[x_{1}, \ldots, x_{n}\right] \mid g(0)=0\right\} \rightarrow \mathbb{R}$ extends to

$$
{ }^{*} \mathrm{fpt}_{0}:\left\{F \in{ }^{*}\left(k\left[x_{1}, \ldots, x_{n}\right]\right) \mid F(0)=0\right\} \rightarrow{ }^{*} \mathbb{R}
$$

such that ${ }^{*} \mathrm{fpt}_{0}(F)=\left[\mathrm{fpt}_{0}\left(f_{m}\right)\right] \in{ }^{*} \mathbb{R}$.

It is a general fact that for every element $w \in{ }^{*} \mathbb{R}$ there is a unique real number, its shadow denoted by $\operatorname{sh}(w)$ such that $|w-\operatorname{sh}(w)|<\varepsilon$ for every positive real number $\varepsilon$ (see Gol, $§ 5.6$ ). Moreover, Theorem 6.1 in loc. cit. implies that if $c_{m}$ is a sequence of real numbers converging to $c$, then $\operatorname{sh}\left(\left[c_{m}\right]\right)=c$. Hence in order to conclude we need to show that $\operatorname{sh}\left({ }^{*} \mathrm{fpt}_{0}(F)\right)=\operatorname{fpt}(f)$. Denoting by $\left(\_\right)^{\circ}$ the subsets consisting of polynomials or power series vanishing at zero, this means that we have to show that the following diagram commutes:

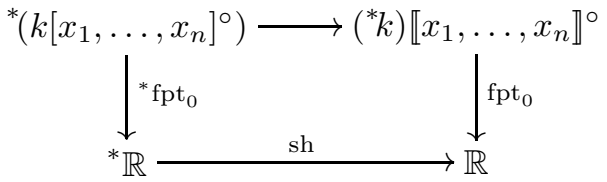


For every positive integer $d$, we denote by $f_{\leq d}$ the truncation of $f$ of degree $\leq d$. It follows from Corollary 3.4 that

$$
\left|\operatorname{fpt}(f)-\operatorname{fpt}\left(f_{\leq d}\right)\right| \leq \frac{n}{d+1} .
$$

We have by definition $f_{\leq d}=\left[\left(f_{m}\right)_{\leq d}\right]$; hence

$$
\left|{ }^{*} \operatorname{fpt}_{0}(F)-{ }^{*} \operatorname{fpt}_{0}\left(f_{\leq d}\right)\right|=\left[\left|\operatorname{fpt}_{0}\left(f_{m}\right)-\operatorname{fpt}_{0}\left(\left(f_{m}\right)_{\leq d}\right)\right|\right] \leq n /(d+1) .
$$

If we show that $\operatorname{fpt}\left(f_{\leq d}\right)={ }^{*} \mathrm{fpt}_{0}\left(f_{\leq d}\right)$, then we are done. Indeed, we deduce from (41) and (5) that $\left|\operatorname{fpt}(f)-{ }^{*} \operatorname{fpt}_{0}(F)\right| \leq 2 n /(d+1)$ for any $d$. Since $\operatorname{fpt}(f) \in \mathbb{R}$, this implies $\operatorname{fpt}(f)=\operatorname{sh}\left({ }^{*} \mathrm{fpt}_{0}(F)\right)$.

To simplify the notation we put $g_{m}=\left(f_{m}\right)_{<d}$ and $g=\left[g_{m}\right]$. It follows from Proposition 3.2 that for any polynomial $h \in k\left[x_{1}, \ldots, x_{n}\right]$ with $h(0)=0$, we may compute $\mathrm{fpt}_{0}(h)$ by considering $h$ in $\left(k^{*}\right) \llbracket x_{1}, \ldots, x_{n} \rrbracket$.

Recall that if we bound the degree of a polynomial, then we can also bound the denominators of its $F$-jumping exponents, independent of the base field (see Proposition 3.6 in $[\mathrm{BMS}]$ ). Since the $F$-pure thresholds of principal ideals are bounded above by 1 , it follows that there is a finite set of rational numbers $A$ such that $\operatorname{fpt}(g) \in A$ and $\operatorname{fpt}\left(g_{m}\right) \in A$ for every $m$. This implies that there is a unique $a \in A$ such that $\left\{m \in \mathbb{N} \mid \operatorname{fpt}\left(g_{m}\right)=a\right\} \in \mathcal{U}$.

Let us prove for example that $a \leq \operatorname{fpt}(g)$ (the reverse inequality follows by an analogous argument). We choose positive integers $r$ and $e$ such that $a \geq \frac{r+1}{p^{e}}$ and every element in $A$ that is $<a$ is also $<\frac{r}{p^{e}}$. Since $\operatorname{fpt}(g) \in A$, if we show that $g^{r} \notin\left(x_{1}^{p^{e}}, \ldots, x_{n}^{p^{e}}\right)$, then $\operatorname{fpt}(g) \geq \frac{r}{p^{e}} ; \operatorname{hence} \operatorname{fpt}(g) \geq a$. Note that if for some $m$ we have $\operatorname{fpt}\left(g_{m}\right)=a$, then using Remark 3.1 we get $g_{m}^{r} \notin\left(x_{1}^{p^{e}}, \ldots, x_{n}^{p^{e}}\right)$. Hence there is a monomial $x_{1}^{b_{1}} \cdots x_{n}^{b_{n}}$ with all $b_{i} \leq p^{e}-1$ that does not appear in $g_{m}$. Note that the set of those $m$ that satisfy this condition is in $\mathcal{U}$. Since there are only finitely many monomials as above, it follows that after possibly passing to a smaller subset we may assume in addition that the same monomial works for all these $m$. This means that the coefficient of the monomial in $g^{r}=\left[g_{m}^{r}\right]$ is nonzero; hence $g^{r} \notin\left(x_{1}^{p^{e}}, \ldots, x_{n}^{p^{e}}\right)$, as required. This proves that $\mathcal{T}_{n}$ is closed.

The last assertion in the theorem follows since $\mathcal{T}_{n}$ is contained in $\mathbb{Q}$ by Theorem 1.1 .

\section{REMARKS AND OPEN PROBLEMS}

Recall that test ideals satisfy the following analogue of the Subadditivity Theorem for multiplier ideals. If $\mathfrak{a}$ and $\mathfrak{b}$ are ideals in $R$ and if $\lambda \in \mathbb{R}_{+}$, then

$$
\tau\left((\mathfrak{a} \mathfrak{b})^{\lambda}\right) \subseteq \tau\left(\mathfrak{a}^{\lambda}\right) \cdot \tau\left(\mathfrak{b}^{\lambda}\right) .
$$

See, for example, Lemma 2.10 in BMS for a proof. In the case of a $p$-power, we have the following strengthening.

Proposition 4.1. If $\mathfrak{a}$ is an ideal in $\mathbb{R}_{+}$and if $\lambda \in \mathbb{R}_{+}$, then

$$
\tau\left(\mathfrak{a}^{p \lambda}\right) \subseteq \tau\left(\mathfrak{a}^{\lambda}\right)^{[p]} .
$$

Moreover, if $\tau\left(\mathfrak{a}^{p \lambda}\right) \subseteq J^{[p]}$ for some ideal $J$, then $\tau\left(\mathfrak{a}^{\lambda}\right) \subseteq J$. 
Proof. If $e \gg 0$, then $\tau\left(\mathfrak{a}^{\lambda}\right)=\left(\mathfrak{a}^{\left\lceil\lambda p^{e+1}\right\rceil}\right)^{\left[1 / p^{e+1}\right]}$ and $\tau\left(\mathfrak{a}^{p \lambda}\right)=\left(\mathfrak{a}^{\left\lceil\lambda p^{e+1}\right\rceil}\right)^{\left[1 / p^{e}\right]}$. By definition we have

$$
\mathfrak{a}^{\left\lceil\lambda p^{e+1}\right\rceil} \subseteq\left(\left(\left(\mathfrak{a}^{\left\lceil\lambda p^{e+1}\right\rceil}\right)^{\left[1 / p^{e+1}\right]}\right)^{[p]}\right)^{\left[p^{e}\right]}
$$

hence $\left(\mathfrak{a}^{\left\lceil\lambda p^{e+1}\right\rceil}\right)^{\left[1 / p^{e}\right]} \subseteq\left(\left(\mathfrak{a}^{\left\lceil\lambda p^{e+1}\right\rceil}\right)^{\left[1 / p^{e+1}\right]}\right)^{[p]}$, which gives (6) .

Suppose now that

$$
\tau\left(\mathfrak{a}^{p \lambda}\right)=\left(\mathfrak{a}^{\left\lceil\lambda p^{e+1}\right\rceil}\right)^{\left[1 / p^{e}\right]} \subseteq J^{[p]}
$$

It follows that $\mathfrak{a}^{\left\lceil\lambda p^{e+1}\right\rceil} \subseteq\left(J^{[p]}\right)^{\left[p^{e}\right]}=J^{\left[p^{e+1}\right]}$. Therefore $\tau\left(\mathfrak{a}^{\lambda}\right)=\left(\mathfrak{a}^{\left\lceil\lambda p^{e+1}\right\rceil}\right)^{\left[1 / p^{e+1}\right]} \subseteq$ $J$.

Remark 4.2. The above proposition gives another proof for the fact that if $\lambda$ is an $F$-jumping exponent for an ideal $\mathfrak{a}$, then $p \lambda$ is also an $F$-jumping exponent. More precisely, if $\varepsilon>0$ is such that $\tau\left(\mathfrak{a}^{p \lambda-\varepsilon}\right)=\tau\left(\mathfrak{a}^{p \lambda}\right)$, then $\tau\left(\mathfrak{a}^{\lambda-\frac{\varepsilon}{p}}\right)=\tau\left(\mathfrak{a}^{\lambda}\right)$.

As we have already mentioned, there are many analogies between the $F$-pure threshold and a characteristic zero invariant that is very much studied, the log canonical threshold (see [TW] and also [MTW]). However, in characteristic zero there is not much difference in considering log canonical thresholds of principal or of arbitrary ideals. This is not the case in characteristic $p$. For example, every rational number $c$ is equal to $\operatorname{fpt}(\mathfrak{a})$ for some ideal $\mathfrak{a}$ in some polynomial ring $R$ : if $c=\frac{n}{r}$, we may take $R=k\left[x_{1}, \ldots, x_{n}\right]$ and $\mathfrak{a}=\left(x_{1}, \ldots, x_{n}\right)^{r}$. On the other hand, as the following proposition shows, there are intervals in $(0,1)$ containing no $F$-pure threshold of a principal ideal in any dimension. For example, there is no such $F$-pure threshold in $\left(1-\frac{1}{p}, 1\right)$. The proposition also follows from the results in Section 2 (see Remark 2.6), but we give a direct argument below.

Proposition 4.3. Let $R$ be a regular $F$-finite ring of characteristic $p$, and $f \in R$.

(i) Let $\alpha=\frac{r}{p^{e}-1}$ for some positive $r$ and $e$, and we put $\alpha_{m}=\left(1-\frac{1}{p^{m e}}\right) \alpha$ for $m \geq 0$. If there is an $F$-jumping exponent of $f$ in $\left(\alpha_{m+1}, \alpha_{m+2}\right]$, then there is an $F$-jumping exponent of $f$ also in $\left(\alpha_{m}, \alpha_{m+1}\right]$.

(ii) For every $e \geq 1$ and every $0 \leq a \leq p^{e}-1$, the $F$-pure threshold $\operatorname{fpt}(f)$ does not lie in $\left(\frac{a}{p^{e}}, \frac{a}{p^{e}-1}\right)$.

Note that (ii) gives for every $e$ open intervals of total length

$$
\sum_{0 \leq i \leq p^{e}-1} i / p^{e}\left(p^{e}-1\right)=1 / 2
$$

containing no $F$-pure threshold of a principal ideal in characteristic $p$. One should compare this with the characteristic zero case when every $c \in(0,1]$ is the $\log$ canonical threshold of some hypersurface: for example, if $c=\frac{n}{r} \leq 1$, then $c$ is the $\log$ canonical threshold of $\sum_{i=1}^{n} x_{i}^{r}$.

Proof of Proposition 4.3. The assertion in (i) follows from the fact that if $\lambda \in$ $\left(\alpha_{m+1}, \alpha_{m+2}\right]$, then $p^{e} \lambda-r \in\left(\alpha_{m}, \alpha_{m+1}\right]$, and we have seen that $p^{e} \lambda-r$ is an $F$-jumping exponent if $\lambda$ is as well. In particular, we see that if $\lambda$ is an $F$-jumping 
exponent in $\left(\alpha_{1}, \alpha\right)$, then there is another positive $F$-jumping exponent $<\lambda$. Hence $\lambda$ is not the $F$-pure threshold of $f$.

Motivated by the analogy with some important conjectures on log canonical thresholds in characteristic zero (see [Kol], §8) we make the following conjectures on $F$-pure thresholds.

Conjecture 4.4. For every prime $p$ and every $n$, the set $\mathcal{T}_{n}$ satisfies $A C C$ (the Ascending Chain Condition), i.e. it contains no strictly increasing sequences.

Remark 4.5. Note that if $f \in k \llbracket x_{1}, \ldots, x_{n} \rrbracket$ lies in the maximal ideal, then Corollary 3.4 implies that whenever $f-g \in\left(x_{1}, \ldots, x_{n}\right)^{d}$, we have $|\operatorname{fpt}(f)-\operatorname{fpt}(g)| \leq$ $n / d$. Therefore the above conjecture predicts that given $f$, there is $d$ such that $\operatorname{fpt}(f) \geq \operatorname{fpt}(f+h)$ for all $h \in\left(x_{1}, \ldots, x_{n}\right)^{d}$. However, even this special case is not known.

Conjecture 4.6. For every prime $p$ and every $n \geq 1$, the accumulation points of $\mathcal{T}_{n}$ are contained in $\mathcal{T}_{n-1}$ (by convention, $\left.\mathcal{T}_{0}=\{0\}\right)$.

We have seen in the previous section that the set $\mathcal{T}_{n}$ is the closure of $\mathcal{T}_{n}^{\circ}$. In fact, we make also the following conjecture.

Conjecture 4.7. For every prime $p$ and every $n$, every $F$-pure threshold in dimension $\leq n$ can be obtained as the F-pure threshold at the origin of some polynomial in $k\left[x_{1}, \ldots, x_{n}\right]$, i.e. we have $\mathcal{T}_{n}=\mathcal{T}_{n}^{\circ}$.

\section{ACKNOWLEDGEMENTS}

The second author is indebted to Caucher Birkar for explaining the usefulness of nonstandard methods. He would also like to thank the Institute for Advanced Study, where part of this work was carried out.

\section{REFERENCES}

[AMBL] J. Alvarez-Montaner, M. Blickle and G. Lyubeznik, Generators of $D$-modules in positive characteristic, Math. Res. Lett. 12 (2005), 459-473. MR2155224 (2006m:13024)

[Bli] M. Blickle, The intersection homology $D$-module in finite characteristic, Ph.D. thesis, University of Michigan, 2001, math. AG/0110244.

[BMS] M. Blickle, M. Mustaţă and K. E. Smith, Discreteness and rationality of $F$-thresholds, Michigan Math. J. 57 (2008), 43-61.

[dFM] T. de Fernex and M. Mustaţă, Limits of log canonical thresholds, Ann. Sci. École Norm. Sup., to appear.

[Gol] R. Goldblatt, Lectures on the hyperreals. An introduction to nonstandard analysis. Graduate Texts in Mathematics 188, Springer-Verlag, New York, 1998. MR.1643950 (2000a:03113)

[Ha] N. Hara, with an appendix by P. Monsky, $F$-pure thresholds and $F$-jumping coefficients in dimension two, Math. Res. Lett. 13 (2006), 747-760. MR2280772 (2007m:14032)

[HY] N. Hara and K.-i. Yoshida, A generalization of tight closure and multiplier ideals, Trans. Amer. Math. Soc. 355 (2003), 3143-3174. MR.1974679 (2004i:13003)

[Kol] J. Kollár, Singularities of pairs, in Algebraic geometry, Santa Cruz 1995, 221-286, volume 62 of Proc. Symp. Pure Math., Amer. Math. Soc., 1997. MR1492525 (99m:14033)

[Laz] R. Lazarsfeld, Positivity in algebraic geometry II, Ergebnisse der Mathematik und ihrer Grenzgebiete, vol. 49, Springer-Verlag, Berlin, 2004. MR.2095472 (2005k:14001b)

[Lyu] G. Lyubeznik, $F$-modules: applications to local cohomology and $D$-modules in characteristic $p>0$, J. Reine Angew. Math. 491 (1997), 65-130. MR1476089 (99c:13005) 
[MTW] M. Mustaţă, S. Takagi and K.-i. Watanabe, F-thresholds and Bernstein-Sato polynomials, European Congress of Mathematics, 341-364, Eur. Math. Soc., Zürich, 2005. MR.2185754 (2007b:13010)

[Ta1] S. Takagi, Formulas for multiplier ideals on singular varieties, Amer. J. Math. 128 (2006), 1345-1362. MR2275023(2007i:14006)

[Ta2] S. Takagi, Adjoint ideals along closed subvarieties of higher codimension, J. Reine Angew. Math., to appear.

[TW] S. Takagi and K.-i. Watanabe, On F-pure thresholds, J. Algebra 282 (2004), 278-297. MR2097584(2006a:13010)

Fachbereich Mathematik, Universität Duisburg-Essen, Standort Essen, 45117 Essen, GERMANY

E-mail address: manuel.blickle@uni-essen.de

Department of Mathematics, University of Michigan, Ann Arbor, Michigan 48109

E-mail address: mmustata@umich.edu

Department of Mathematics, University of Michigan, Ann Arbor, Michigan 48109

E-mail address: kesmith@umich.edu 